\title{
Rate Constant
}

National Cancer Institute

\section{Source}

National Cancer Institute. Rate Constant. NCI Thesaurus. Code C94967.

Parameter appearing in models to describe a unidirectional transfer rate out of a compartment. It is the (amount transferred per unit time)/(amount present in the compartment). 